This item was submitted to Loughborough's Research Repository by the author.

Items in Figshare are protected by copyright, with all rights reserved, unless otherwise indicated.

\title{
Inference of missing PV monitoring data using neural networks
}

PLEASE CITE THE PUBLISHED VERSION

https://doi.org/10.1109/PVSC.2016.7750305

PUBLISHER

(c) IEEE

VERSION

AM (Accepted Manuscript)

LICENCE

CC BY-NC-ND 4.0

REPOSITORY RECORD

Koumpli, Elena, Diane Palmer, Thomas R. Betts, Paul Rowley, and Ralph Gottschalg. 2019. "Inference of Missing PV Monitoring Data Using Neural Networks". figshare. https://hdl.handle.net/2134/27501. 


\title{
Inference of Missing PV Monitoring Data using Neural Networks
}

\author{
Eleni Koubli, Diane Palmer, Tom Betts, Paul Rowley and Ralph Gottschalg \\ Centre for Renewable Energy Systems Technology (CREST) \\ Loughborough University, Loughborough, Leicestershire, LE11 3TU, United Kingdom
}

\begin{abstract}
Complete photovoltaic monitoring data are required in order to evaluate PV system performance and to ensure confidence in project financing. Monitoring sub-system failures are common occurrences, reducing data availability in meteorological and electrical datasets. A reliable backfilling method can be applied in order to mitigate the impact of long monitoring gaps on system state and performance assessment. This paper introduces a method of inferring in-plane irradiation from remotely obtained global horizontal irradiation, by means of a neural network approach. Generation output is then calculated utilizing a simple electrical model with fitted coefficients. The proposed method is applied to a UK case study for which the mean absolute error in monthly system output was reduced significantly, to as low as $0.9 \%$. This yielded more accurate results in backfilling the missing datasets when compared to standard approaches. The impact of missing data on monthly performance ratio is also investigated. Using backfilling to synthesize lost data increases performance ratio prediction accuracy significantly when compared to simply omitting such periods from the calculation.

Index terms - missing data, monitoring data, neural networks, solar radiation data, photovoltaic (PV) systems
\end{abstract}

\section{INTRODUCTION}

The assessment of PV system performance requires the acquisition of meteorological and electrical data during system operation. Complete data from the monitoring systems are required to evaluate overall system performance for a given time period, without introducing bias caused by omitting longer gaps (a day, few days or even a month). However, in realistic situations data availability is seldom $100 \%$. This is important since incomplete datasets reduce confidence in assessments of the system operational state and performance ratio, on which preferential project financing is often dependent. Furthermore, lack of monitoring data at sub-system level obscures fault detection and increases the risk of revenue loss. Thus, the aim of data backfilling is to minimise potential risks deriving from monitoring failures by inferring lost information with the highest possible accuracy.

It is important that every performance analysis is based upon an accurate and uninterrupted dataset. In some cases if data availability is less than $10 \%$ then performance indicators cannot be accurately evaluated [1]. Backfilling strategies so far employed are varied, but there are no standardized or accepted methods to date. It is also likely that the accuracy of backfilling depends upon various performance factors such as PV system component degradation, seasonal variation and weather variability. This paper presents a backfilling method which is validated against a realistic case of data loss and takes into account weather and PV system specific variables.
It is possible to backfill missing meteorological and/or electrical data using synthetic climatic data and/or electrical readings using past available data from the monitoring system and achieve good accuracy on monthly and annual performance ratio (PR) [2],[3]. Specifically, the second case in [3] describes an interrupted monitoring system, where both climatic and electrical readings are missing for a month. In this case, the required datasets were acquired with the methods also briefly discussed here, giving satisfactory results for performance ratio. However, bias deriving from inferring in-plane irradiation caused a noticeable underestimation in energy yield. This was found to be due to the separation and translation algorithms employed to translate global horizontal irradiation to in-plane. Relevant results to this effect are discussed in [4].

The work described here improves the method accuracy through reducing the bias in modelled in-plane irradiation by means of artificial neural networks (NN). This is done by utilizing past available data from the monitoring system and sun position as inputs into the $\mathrm{NN}$ training set. Two different training sets were tested to determine the most suitable for the case study. A significant reduction in bias (quantified as mean bias error or MBE) in irradiation was observed. This delivers a significant improvement in the accuracy of the energy yield assessment.

Neural networks have been used previously in photovoltaic modelling to predict energy yield (e.g. see [5]), in solar irradiance forecasting [6] and to translate global horizontal irradiance (GHI) to in-plane for specific climate and angles [7]. This last application could be of little practical interest if past data are not available for a PV system's location. However, in the context of backfilling where data exist except for a specific period only, this is an application of NN that gains merit as it completely omits the two steps of irradiance separation and translation algorithms which contribute the most to error in estimated yield. Moreover, simple NN training sets are proposed which exploit as few input data as possible, increasing the method's applicability.

\section{DESCRIPTION OF THE METHOD}

\section{A. Overview of the steps}

For the examined case of missing data, energy output readings of a c-Si module and a concurrent climatic dataset are utilized. The data are taken from CREST's outdoor monitoring system. In order to validate the modelling results (i.e. the backfilling method), a period of one month is artificially removed from the initial dataset and is treated as the "missing" 
TABLE 1

NEURAL NETWORKS TESTED TRAINING SETS

\begin{tabular}{|c|c|c|c|c|}
\hline Training set & \multicolumn{2}{|c|}{ Input variables } & Target \\
\hline Validation & $\begin{array}{c}\text { Measured global } \\
\text { horizontal irradiance } \\
(\mathrm{GHI})\end{array}$ & Azimuth & Zenith & In-plane irradiance \\
\hline Training set a & Measured GHI & Azimuth & Zenith & In-plane irradiance \\
\hline Training set b & Interpolated GHI & Azimuth & Zenith & In-plane irradiance \\
\hline
\end{tabular}

period, while later it is used for comparison with the backfilled results.

To backfill the missing data in the monitoring datasets the steps are the following:

1) Inferring global horizontal irradiance: This is done by applying a spatial interpolation technique (Kriging) to data from a network of about 80 meteorological stations across the UK (see more in [3] and [8]). The time resolution of the data available is hourly. Ambient temperature is also interpolated with this method.

2) Translating to in-plane irradiance: This is normally done by using algorithms for separation into beam and diffuse components (e.g. [9]) and translation to an inclined plane (e.g. [10],[11]). This is referred to as the two-step method. The specific algorithms used here as a baseline were chosen as they have been shown to slightly outperform other models for the location of this study. These two steps may be replaced by using a single-step neural network approach, described in the following section. Module temperature and energy yield can then be calculated by using a thermal and an electrical model respectively, with input data the backfilled in-plane irradiation and ambient temperature.

3) Calculating module temperature: For this step the Ross model is used [12]. The parameter $\mathrm{k}$ is first determined by using experimental data of one year of ambient (Ta) and module temperature $\left(\mathrm{T}_{\mathrm{m}}\right)$ and inplane irradiance $(\mathrm{G})$ :

$$
T_{m}=T_{a}+k \cdot G
$$

4) Calculating energy output: For this step the model described in [13] is used:

$$
\begin{aligned}
& P^{\prime}\left(G^{\prime}, T^{\prime}\right)=G^{\prime}\left(1+k_{1} \ln \left(G^{\prime}\right)+k_{2} \ln \left(G^{\prime}\right)^{2}\right. \\
& \left.+k_{3} T^{\prime}+k T^{\prime} \ln \left(G^{\prime}\right)+k_{5} T^{\prime} \ln \left(G^{\prime}\right)+k_{6} T^{\prime 2}\right)
\end{aligned}
$$

Where $\mathrm{P}^{\prime}=\mathrm{P}_{\mathrm{MP}} / \mathrm{P}_{\mathrm{STC}}, \mathrm{G}^{\prime}=\mathrm{G} / \mathrm{G}_{\mathrm{sTC}}$ and $\mathrm{T}_{\mathrm{m}}{ }^{\prime}=\mathrm{T}_{\mathrm{m}}-\mathrm{T}_{\mathrm{STC}}(\mathrm{STC}=$ Standard Testing Conditions) and $\mathrm{P}_{\mathrm{MP}}$ is maximum power. In this case, the coefficients of the electrical model are obtained by fitting experimental data of power, irradiance and temperature taken from a monitoring period (training period) close to the missing period, as this captures the state of the system and accounts for degradation and other changes (for the training process refer to [3]).

\section{B. Neural Networks}

At this point, only in-plane irradiation is backfilled by means of a neural network. This step has the highest contribution to the uncertainty in yield calculation, in terms of both random and bias errors [4]. The structure of neural networks applied in this work consists of three layers; the input layer, a single hidden layer and the output layer. The output layer consists of the target output, which in this case is in-plane irradiation. The input layer consists of an array of variables, the training set, comprising global horizontal irradiation and two sun position angles. TABLE 1 describes the training sets that have been used. A neural network package for the Python programming language [14] was used for the implementation of the network, particularly choosing a back-propagation algorithm for the training process and Levenberg-Marquardt optimization algorithm, both suitable for problems of relatively low complexity.

The training set used here aims to rely on as little information as possible, given that only global horizontal irradiation is usually available from the met stations. Solar azimuth and zenith angles are calculated using a solar position algorithm [15]. Different neuron configurations were tested as well as number of repetitions. Using one hidden layer with a size of 5 neurons and 1000 repetitions yielded satisfactory results based on the coefficient of determination $\left(R^{2}=0.9938\right)$, while adding further neurons and repetitions from that point increased computational capacity without significantly affecting the result.

The procedure of training and modelling with NN goes as follows: First, the data set is divided to the training and the testing (validation) set. In this work, a year's worth of data was utilized where 1 month was used as the testing period and the remainder was used for the training. Then, after the training is complete, the network configuration is used to model the output of the testing (or missing) period using the same type of input parameters used for training. For the validation of the method, global horizontal irradiation (GHI) is taken from the monitoring system whereas for backfilling the missing period, GHI is obtained using the method described in section A. For the case 
of backfilling, two different sources of horizontal irradiation were utilized in the training set; measured and interpolated horizontal irradiation. The results of the tested methods are compared by means of the statistical root-mean-square-error (RMSE) and mean-bias-error (MBE) for the following modelled parameters: In-plane irradiation, energy output and performance ratio. The validation results are shown in Fig. 1

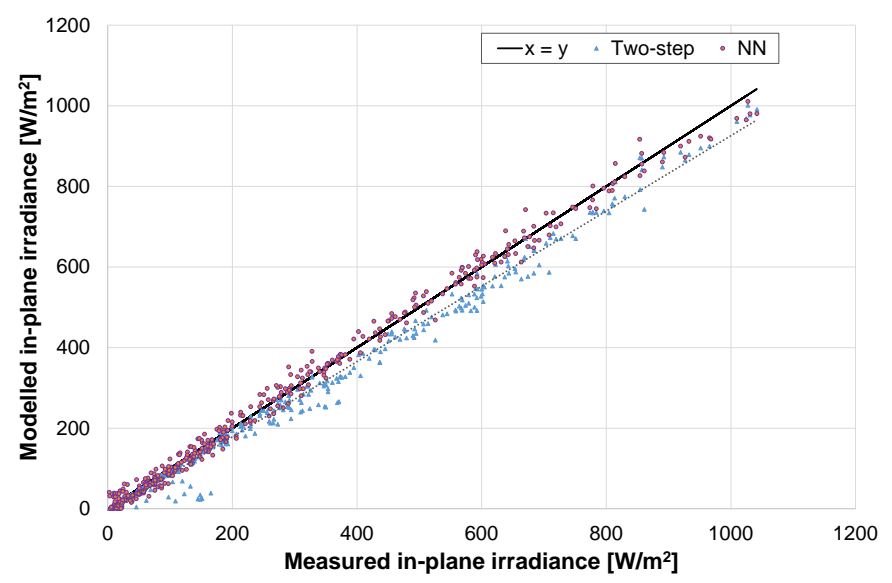

Fig. 1. Comparison of hourly measured and modelled in-plane irradiance using neural networks (NN) and the two-step method (BRL) for the testing period of one month (June).

where the NN method is compared to the two-step method. The hourly \%RMSE and \%MBE were 13.8 and -9.4 for the two-step method, respectively and 7.0 and -0.15 for the NN method. Similar results were obtained for different testing periods. The two-step method consistently underestimates in-plane irradiation in comparison to the NN method, while also giving a slightly higher RMSE. This shows that for a specific orientation and inclination the prediction of in-plane irradiation with a neural network approach shows promising results.

\section{RESULTS}

The next step is to backfill the missing month; so in-plane irradiation is inferred from interpolated global horizontal irradiation using the three approaches above, then energy output and finally the performance ratio (PR) are calculated for each case.

\section{A. In-plane irradiation}

The two-step conventional method and NN for two different training sets, given in TABLE 1, were compared. Training set (a) comprises global horizontal irradiation received from the monitoring system, i.e. it is measured on site and target in-plane irradiation is also taken from the monitoring system. Training set (b) comprises global horizontal irradiation taken from the Kriging method, thereby it is modelled, whereas target in-plane irradiation is taken from the monitoring system as before.

The results for one month are depicted in Fig. 2. The monthly results show that the \%MBE for the two-step method is -5.6 whereas for the NN training sets a, b, is +3.5 and +0.8 respectively, resulting in a significant absolute reduction in MBE for the monthly result in favor of the NN (training b) method.

Both NN methods have a reduced MBE compared to the conventional method. The underestimation of the two-step

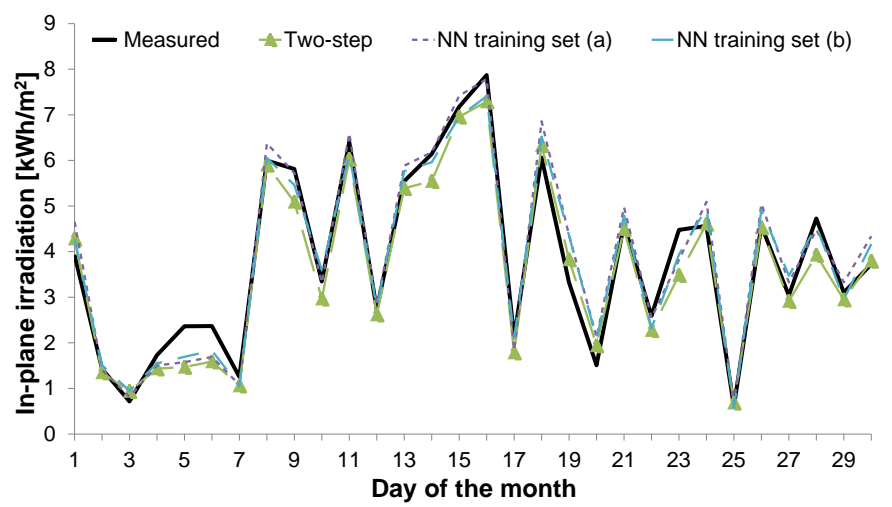

Fig. 2. In-plane irradiation (daily) results obtained with three methods: Two-step and NN using two different training sets (a) and (b) for a missing month (April).

method derives from the algorithms involved for the translation to in-plane irradiance as shown in Fig. 1.

The slight overestimation in the NN methods derives from the small overestimation in global horizontal irradiation from the Kriging interpolation, giving an $\mathrm{MBE}$ of $+3.5 \%$ for the testing month. This seems to have a direct impact on the results obtained using the NN (a) training set, but is not evident in the case of the NN (b) training set. Thus, using interpolated horizontal irradiation as part of the training set eliminates the resulting bias in in-plane irradiation considerably.

\section{B. Energy output and $P R$}

The next step is to use (2) to calculate module temperature and finally (3) to calculate energy output. But firstly, the power model is validated against measured maximum power for the testing month (see Fig. 3). For the validation of the model, hourly measured in-plane irradiation and module temperature were used as input. The same model coefficients which were used in validation were also used for backfilling.

The validation results yielded an hourly \%RMSE and \%MBE of 6.8 and 0.76 , respectively. These figures give an idea of the error deriving from modelling the PV system behavior, which is very low compared to the error contribution from all irradiation modelling steps. The validation procedure was also repeated for up to five "missing" consecutive months yielding an average monthly \%MBE as low as 0.56 .

The next step is to compare the energy output from the three methods used for obtaining in-plane irradiation, the two-step and the two NN methods (a) and (b) for the (same) test month. The daily results are depicted in Fig. 4. 
The monthly \%MBE for the energy output for the two-step method and the NN training sets (a), (b) are $-5.6,+3.1$ and +0.9 , respectively. Again, this is a noticeable absolute reduction in MBE for both NN methods.

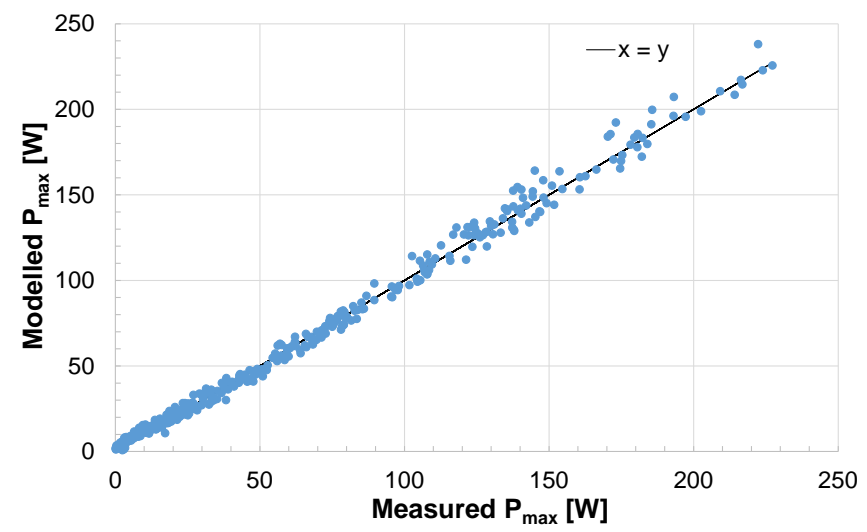

Fig. 3. Comparison of hourly measured and modelled maximum power output using equation (3) and measured input data of in-plane irradiance and module temperature.

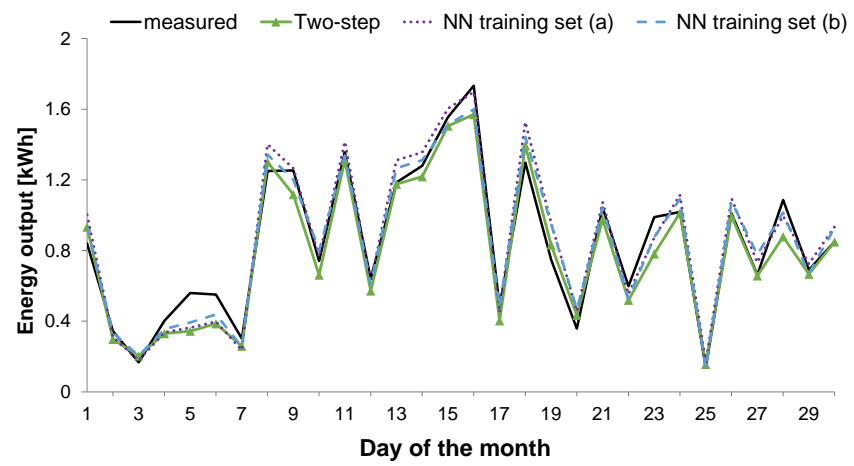

Fig. 4. Energy output results obtained with the two-step and NN methods for two different training sets (a) and (b), for a missing month.

The final step is to calculate the PR with:

$$
\mathrm{PR}=\left(\mathrm{E} \cdot \mathrm{G}_{\mathrm{STC}}\right) /\left(\mathrm{H} \cdot \mathrm{P}_{\mathrm{STC}}\right)
$$

Where $\mathrm{E}$ is the energy output (Wh), $\mathrm{H}$ is the total in-plane irradiation as modelled or measured $\left(\mathrm{Wh} / \mathrm{m}^{2}\right)$ and $\mathrm{G}_{\text {STC }}, \mathrm{P}_{\text {STC }}$ are irradiance and power, respectively, at standard testing conditions (STC). Using (3) the actual PR for the testing month is 0.90 (remembering it is a single module in this case, and DC PR). Using the three methods for modelling the PR also yielded 0.90 for the testing month with $\mathrm{NN}$ (a) method giving the maximum MBE of 0.003 , which nonetheless is much lower than the average deviation of the daily PR (equal to 0.03) throughout the test month. This result is expected, as any bias resulting from in-plane irradiation modelling is propagated through to energy output but is eliminated in the performance ratio, yielding an accurate agreement with the actual PR value.

\section{WHY BACKFILL?}

The applied backfilling method aims to replenish missing or corrupted information on energy output and performance ratio with the lowest errors possible. It can be a useful tool as a means of acquiring data that would normally be available from monitoring, therefore be used in place of monitoring if needed. The main reason for this is that while energy readings are usually available for the total production of a system (AC meter readings or as exported to the grid), if monitoring fails, array or sub-array monitoring data are not available. The proposed method can be applied on array, sub-array or string level and this is especially important for enabling fault detection, thus minimizing downtime.

On the other hand, if irradiation is not available then the energy output alone is not an indicator of normal operation, so in every case the PR on the DC side will have to be calculated, as one of the main indicators in PV performance assessment.

To emphasize the latter case, the impact of missing days in a month on the monthly PR is studied here by artificially removing consequent days from the testing month and looking at the impact on the final result.

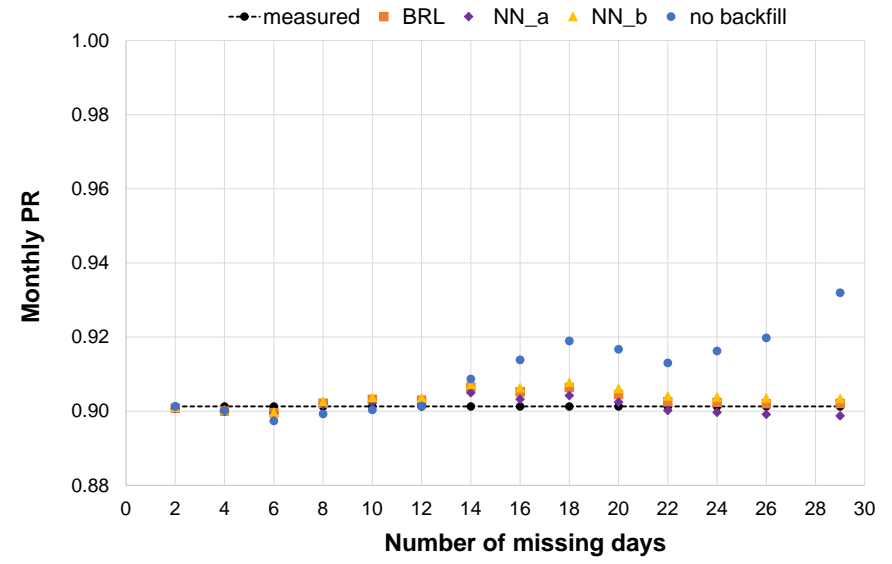

Fig. 5. Impact of the missing days on the monthly PR with backfilled energy output and without.

As illustrated in Fig. 5, after 12 days of missing data, the impact on the PR is more evident if the missing period is not backfilled. While for all the backfilling methods the maximum deviation from the actual PR is as low as 0.007 , the maximum deviation for the case of the non-replenished period is 0.03 which is about $3 \%$ of the monthly PR. In all cases the results show that it is worth backfilling the lost data both for estimating the energy output at sub-system level monitoring as well as for a more accurate prediction of the PR at the end of the month.

Another useful aspect of backfilling, is that it can be used to give an estimation of energy losses caused by total blackouts. In this case, backfilled energy output can be employed to 
estimate energy losses for the period where the fault occurred until it was detected and repaired.

Limitations: There are cases where further considerations should be taken into account prior to backfilling. For example in cases of very prolonged monitoring interruption (for example a year) there is increased probability of PV system faults occurring during the missing period. Under these circumstances, the 'system fingerprinting' implicit in the fitting of the power model coefficients will no longer capture the system operational and behavioral state and actual energy output variations will not be represented accurately with backfilling.

\section{CONCLUSIONS}

A backfilling method for energy output has been presented, where both in-plane irradiation, module temperature and energy output are assumed to be missing (or unusable) using a period of one month as the testing period. In order to infer global horizontal irradiation, an interpolation method has been applied across meteorological stations in the UK. In-plane irradiation, an important modelling parameter, may be acquired by a conventional method using separation and translation algorithms. However, in previous studies, this method has been shown to contribute to an increased negative bias due to the algorithms involved. In this study these algorithms were replaced by means of neural networks and training procedures comprising global horizontal irradiation and sun position angles. The results showed that higher accuracy can be achieved in terms of in-plane irradiation and consequently energy output. In-plane irradiation was inferred for a month showing a \%MBE of 0.8 and energy output with a \%MBE as low as 0.9 , in comparison to the $-5.6 \%$ of the two-step method. The presented approach can be used to replenish missing or incorrect PV system properties such as energy output and performance ratio. Further work has been done on the impact of missing data on the monthly PR and was found that without backfilling, the monthly PR may deviate 3\% from its actual value, whereas using the proposed backfilling methods the obtained PR lies within $0.8 \%$ of its actual value. Therefore, backfilling techniques should be considered for acquiring energy output and a more accurate monthly PR in case of data loss or corruption, as well as estimation of losses due to total blackouts. System failures occurring during the missing period in cases of prolonged interruption of the monitoring system should be examined prior to applying backfilling.

\section{ACKNOWLEDGEMENTS}

This work has been conducted as part of the research project 'PV2025 - Potential Costs and Benefits of Photovoltaic for UK Infrastructure and Society', funded by the RCUK Energy Programme (contract no: EP/K02227X/1). The authors would like to acknowledge the additional support of the European Metrology Research Programme (EMRP) Project ENG55 PhotoClass. The EMRP is jointly funded by the EMRP participating countries within EURAMET and the European Union.

\section{REFERENCES}

[1] S. Kurtz, J. Newmiller, T. Dierauf, A. Kimber, J. Mckee, and R. Flottemesch, "Analysis of Photovoltaic System Energy Performance Evaluation Method," United States. Dept. of Energy, NREL/TP-5200-60628, 2013.

[2] E. Koubli, D. Palmer, P. Rowley, T. R. Betts, and R. Gottschalg, "Assessment of PV System Performance with Incomplete Monitoring Data," in 31st European Photovoltaic Solar Energy Conference and Exhibition, 2015, pp. 15941597.

[3] E. Koubli, D. Palmer, P. Rowley, and R. Gottschalg, "Inference of missing data in photovoltaic monitoring datasets,” IET Renew. Power Gener., vol. 10, no. 4, pp. 434439, 2016.

[4] M. Lave, W. Hayes, A. Pohl, and C. W. Hansen, "Evaluation of Global Horizontal Irradiance to Plane-of-Array Irradiance Models at Locations Across the United States," IEEE J. Photovoltaics, vol. 5, pp. 597-606, 2015.

[5] F. Almonacid, C. Rus, P. Pérez-Higueras, and L. Hontoria, "Calculation of the energy provided by a PV generator. Comparative study: Conventional methods vs. artificial neural networks,” Energy, vol. 36, pp. 375-384, 2011.

[6] A. Mellit and A. M. Pavan, "A 24-h forecast of solar irradiance using artificial neural network: Application for performance prediction of a grid-connected PV plant at Trieste, Italy,” Sol. Energy, vol. 84, pp. 807-821, 2010.

[7] G. Notton, C. Paoli, S. Vasileva, M. L. Nivet, J. L. Canaletti, and C. Cristofari, "Estimation of hourly global solar irradiation on tilted planes from horizontal one using artificial neural networks,” Energy, vol. 39, pp. 166-179, 2012.

[8] P. Rowley, P. Leicester, D. Palmer, P. Westacott, C. Candelise, T. Betts, and R. Gottschalg, "Multi-domain analysis of photovoltaic impacts via integrated spatial and probabilistic modelling," IET Renew. Power Gener., vol. 9, pp. 424-431, 2015.

[9] B. Ridley, J. Boland, and P. Lauret, "Modelling of diffuse solar fraction with multiple predictors," Renew. Energy, vol. 35, pp. 478-483, 2010.

[10] J. E. Hay, R. Perez, and D. C. McKay, “"Estimating Solar Irradiance on Inclined Surfaces: A Review and Assessment of Methodologies,'” Int. J. Sol. Energy, vol. 4, pp. 321-324, 1986.

[11] D. Reindl, W. Beckman, and J. Duffie, "Evaluation of hourly tilted surface radiation models,” Sol. Energy, vol. 45, pp. 917,1990

[12] J. Ross, R. G., "Interface design considerations for terrestrial solar cell modules," in 12th IEEE Photovoltaic Specialist Conference, pp. 801-806, 1976.

[13] T. Huld, G. Friesen, A. Skoczek, R. P. Kenny, T. Sample, M. Field, and E. D. Dunlop, "A power-rating model for crystalline silicon PV modules,” Sol. Energy Mater. Sol. Cells, vol. 95, pp. 3359-3369, 2011.

[14] "Pyrenn: A Recurrent Neural Network Toolbox for Python and Matlab ", 2016. [Online]. Available: http:// pyrenn.read thedocs.io/en/latest [Accessed: 10-Apr-2016].

[15] I. Reda and A. Andreas, "Solar position algorithm for solar radiation applications," Sol. Energy, vol. 76, no. 5, pp. 577589, 2004. 